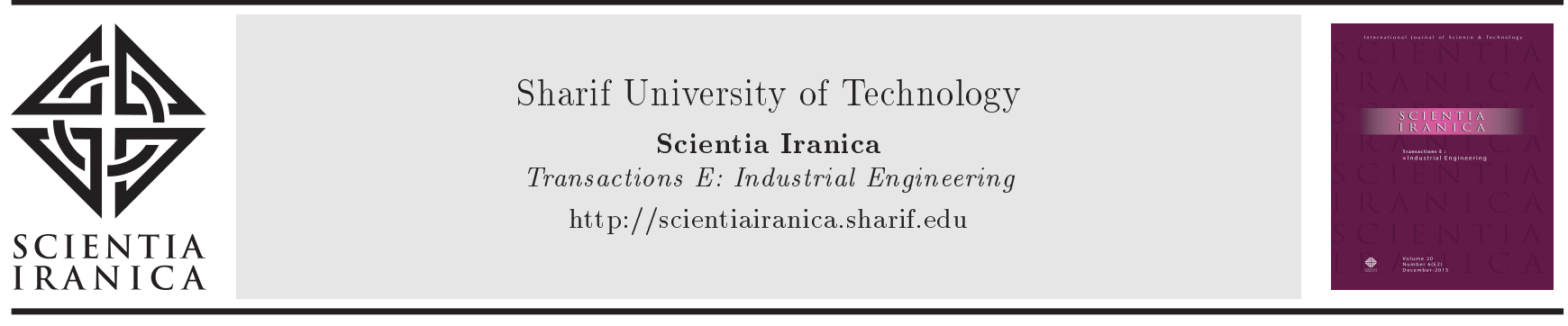

\title{
Resilient network design in a location-allocation problem with multi-level facility hardening
}

\author{
Z. Esfandiyari ${ }^{\mathrm{a}}$, M. Bashiri ${ }^{\mathrm{b}, *}$, and R. Tavakkoli-Moghaddam ${ }^{\mathrm{c}, \mathrm{d}}$ \\ a. Department of Industrial Engineering, Science and Research Branch, Islamic Azad University, Tehran, Iran. \\ b. Department of Industrial Engineering, Shahed University, Tehran, Iran. \\ c. School of Industrial Engineering, College of Engineering, University of Tehran, Tehran, Iran. \\ d. Arts et Metiers ParisTech, LCFC, Metz, France.
}

Received 19 April 2017; received in revised form 28 October 2017; accepted 30 June 2018

\section{KEYWORDS}

Static resilience;

Location-allocation;

Random disruption;

Multi-level hardening;

Lagrangian

decomposition

algorithm.

\begin{abstract}
Many sources of risk affect network elements, which may lead to network failure; thus, planners need to consider them in the network design. One of the most important strategies for disruption risk management is the static resilience. In this strategy, the network functionality is maintained after the disruption event by the prevention and hardening actions. In this paper, a resilient capacitated fixed-charge location-allocation model is proposed. Both facility hardening and equipping of the network with backup facilities for disrupted elements are considered together to avoid supply network failure due to random disruption. Facilities are decided to be hardened in multiple levels before disruption events. The problem is formulated as a non-linear integer programming model; then, its equivalent linear form is presented. A Lagrangian Decomposition Algorithm (LDA) is developed to solve large-scale instances. Computational results confirm the high efficacy of the proposed solution approach, compared to classical solution approaches, in dealing with large-scale problems. Moreover, the superiority of the proposed model is confirmed in comparison to the classical models.
\end{abstract}

(C) 2019 Sharif University of Technology. All rights reserved.

\section{Introduction}

\subsection{Motivation}

Supply networks (e.g., supply chains and electrical power networks) are widespread throughout the world. Such networks consist of a set of facilities (e.g., power substations, ports, and distribution centers) and a set of customers to be served by the facilities. These facilities form the backbone of networks, and their

\footnotetext{
*. Corresponding author.

E-mail addresses: zahra_esfandiyari62@yahoo.com (Z.

Esfandiyari); bashiri@shahed.ac.ir (M. Bashiri);

tavakoli@ut.ac.ir (R.Tavakkoli-Moghaddam)
}

failure may result in interruptions in the flow of goods or services. One common assumption about traditional supply network design decisions is that facilities are always operational and never fail while operating; hence, the possibility of failure is not incorporated into the problem modeling. Because the operation of networks can be disrupted randomly or intentionally, there is a need to perceive how network systems and their performance can be affected by the failure of their facilities in the real-world cases of disruption events. For example, the failure or decline of power supply facilities eventuates in dramatic consequences for a national society and its economy; thus, these facilities are vital entities. The relevance of a continuous power supply to our daily life, industrial production, and electronics stimulates today's society to be highly 
vulnerable and susceptible to power supply stoppage. In all countries, even a short power outage is not acceptable due to having significant impact on the entire economy. For example, power outages in the North East of the United States in 2003 brought adverse effects on business in this area. Due to a labor strike in 2002, 29 ports on the eastern coast of the United States were disabled, leading to the closure of the New United Motor Manufacturing Plant. Natural disasters can also have significant impact on these network performances and their functionalities. In 2010, 450 natural disasters were recorded across the world; in 2011, only the U.S. rescued economic damage worth over 55 billion dollars from natural disasters [1]. After Hurricane Katrina, due to the loss of electric power at the pumping stations, the supply of petroleum products was interrupted. The incidence of these disruptions and their consequences have increased recently. A research center for communicable disasters has stated that disruptions have increased exponentially around the world during the past few decades.

Additionally, facility disruption is sometimes even more important; for instance, failures of emergency rooms in hospitals and Automated Teller Machines (ATMs) may lead to irreparable consequences. These instances and their severe consequences demonstrate that the classic supply network design is not able to cope with disruption by unpredictable events. It has forced decision-makers to consider the possibility of facility disruptions and to protect such facilities from failure during the network design.

\subsection{Related studies}

In the literature, supply network disruption risks are divided into two categories based on their sources: random and intentional disruption risks. The former may occur at any point of the supply network, such as natural hazards (e.g., earthquakes, system component breakdown and plant fire). However, in the latter category, components whose failure may bring maximum damage to a supply network are highly probable to be targeted (e.g., terrorist attacks and labor union strikes) [2].

The reliability of supply networks can be improved by implementing some risk management strategies to mitigate disruption effects. Static resilience is one of the most important strategies of disruption risk management in the supply chain network design. The main objective of this strategy is to maintain the network functionality after disruption and often focuses on reduction options in terms of prevention and protection in order to design reliable systems and prevent (or absorb) adverse events. Facility hardening with additional investment in location-allocation decisions about hedging network from failures is an instance of this strategy that leads to the creation of a reliable and efficient network. Hardened facilities are immune to a failure and do not lose the capacity after disruptive events; thus, they are completely reliable. Facility hardening includes acquiring and installing protective measures, procuring and maintaining backup inventory, and hiring extra workers [3]. Telecommunication networks, water supply, power supply, power distribution, energy networks, etc. are some instances for these strategy applications.

In this paper, we study a facility locationallocation problem with the possibility of facility disruption, considering facility hardening and facility backup for network reliability improvement. The two main categories of studies can be identified in facility hardening for network reliability improvement. In the first one, network facilities are commonly subject to interdiction (i.e., intentional and planned attacks), and decisions are made using bi-level or tri-level game theory models. In such studies, decisions about allocation of protection resources are made to protect networks with facilities that are more effective. The previous studies in [4-8] are examples of the first category. In the second one, facilities face random disruption; there are commonly two types of facilities: unreliable facilities with specific failure probabilities and hardened or reliable ones that must be located. The model makes decisions about facility location and allocation to customers to minimize the total expected cost. The previously published works in [9-12] are some studies in the second category.

To the best of the authors' knowledge, there are only four studies of facility hardening at different levels. Azad et al. [2] and Fattahi et al. [13] classified facilities into different hardening levels and costs; the model makes decisions about locations and the primary and backup assignments [2,13]. However, Jabbarzade et al. [14] considered a model to make a decision about these classified facility locations, customer allocations, inventory, and a number of transshipments from reliable facilities to unreliable ones. Losada et al. [15] found a relationship between network recovery time and different initial amounts of protection investment for different hardening levels. In order to minimize the total expected costs, the model makes decisions on only facility protection investments. In addition, all previous studies have considered a single allocation situation for customers assigned to located facilities via binary decision variables, while the present paper proposes a non-linear integer programming model that considers multiple allocation situations for customers assigned to facilities and facility hardening at different levels. Facilities can be partially hardened under different initial rates of protection investment; therefore, facilities may lose some of their capacity after disruption events based on their hardening levels. Hardened facilities at level one will not lose their capacity after disruption events, 
Table 1. Brief review of studies in facility location for the network reliability improvement.

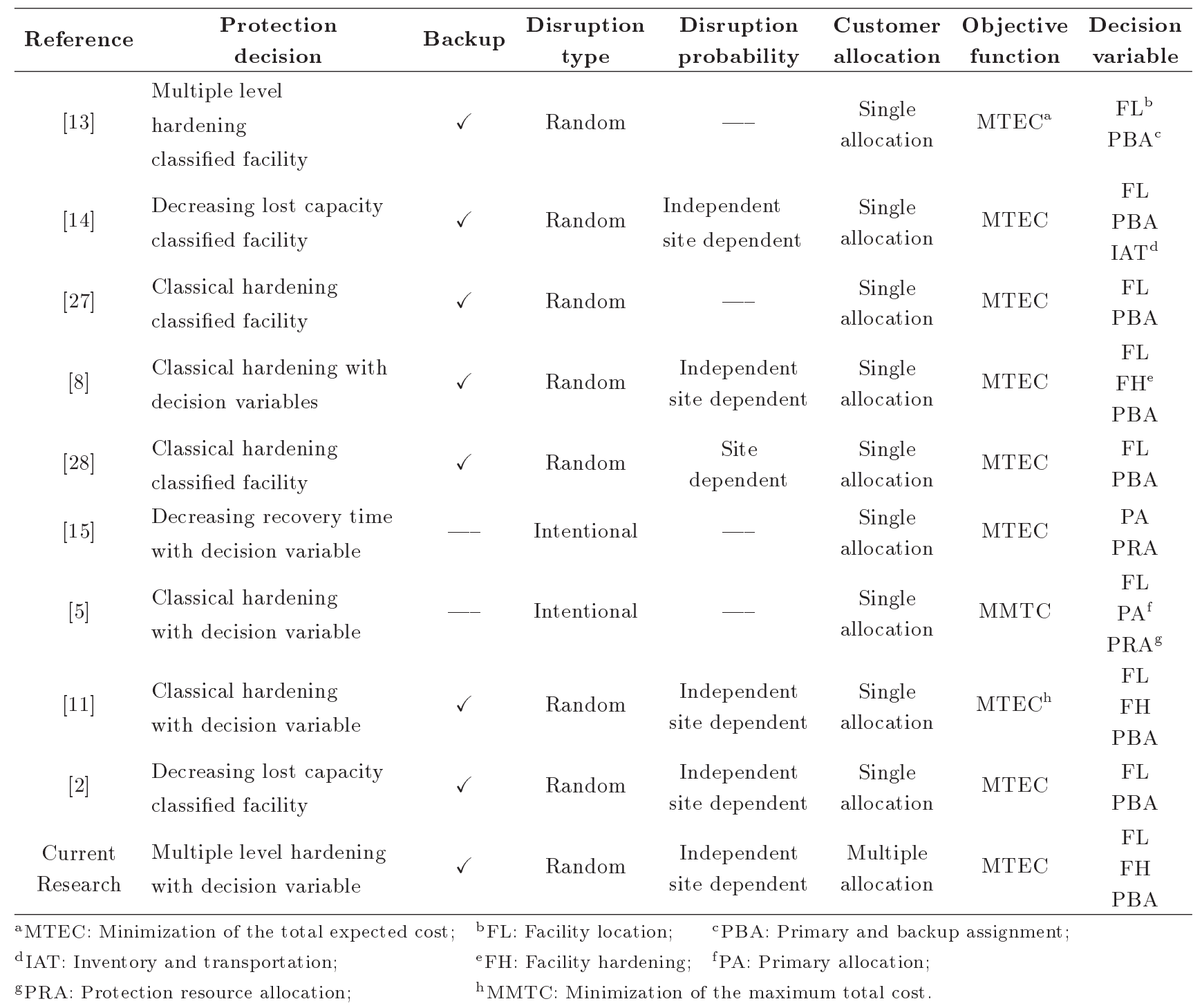

while hardened facilities at level two will lose half of their capacity after such events. Table 1 presents a brief review of the second category-related studies.

One of the real applications of the proposed model is the decisions concerning the protection of hospitals and clinical services against accidental electric power outage during their operation. Different types of medical equipment (e.g., oxygen compressors, dialysis machines, mechanical ventilation, and cardiac monitors) require sufficient power to carry out vital support tasks for patients in these centers. Due to the sensitivity of this equipment to power, the power outages can affect the quality of patient care. Therefore, planners should invest in electricity supply and outage prevention when they construct hospitals and other health care centers, such as clinics, laboratories, and nursing homes [16]. For this purpose, different types of power generators can be used to supply part of the electricity consumed by specific departments in hospitals, such as the emergency rooms in an accidental power outage. In other words, using generators of uninterruptible power of low to moderate power, these units will be only responsive to the electricity consumed by specific departments, such as emergency rooms. Moreover, in order to supply all parts of the hospital in a major electricity disruption, parallel high-capacity power lines can be used. The hardening of the distribution centers against fire in the supply chain design is another application of the proposed model. Fire in these centers can be prevented through maintenance of electrical wiring and accessories, education on the basis of electrical safety principles, and investment in low-risk firefighting equipment. Furthermore, the installation of fire detection systems and rainwater systems will reduce the impact of fire [14]. In this condition, by storing some stocks in these centers, part of customer's demands can be satisfied, and the remaining demands will be supplied by other centers. 
The proposed model can be used in various real case applications, such as two mentioned ones, to have a resilient network.

The main contributions of this study that differentiate it from the past studies in the related literature can be summarized as follows:

- A non-linear integer programming model is developed for the facility location-allocation problem in the supply chain network design, considering a multi-allocation situation for customers assigned to facilities, capacity limitation, and multi-level hardening to optimize the total expected cost in disruption events;

- A comparison of the model priority is made with classical hardening decisions in facility location problems in different facility failure probabilities;

- The proposed model is linearized by suitable techniques;

- A Lagrangian Decomposition Algorithm (LDA) is developed to obtain a solution to large-scale problems in a reasonable amount of time.

The structure of this paper is organized as follows. The proposed model and its proper LDA are presented in Section 2. Section 3 presents the computational results. Finally, results and suggestions for the future research are given in the last section.

\section{Problem description}

The proposed model considers the fixed-charge facility location problem with site-specific facility failure probabilities and facility hardening. The model seeks to minimize the total expected cost by optimally opening facilities, deciding on the hardening level of opened facilities, and allocating customers to them as primary and backup assignments. We describe the assumptions, notations, and parameters to formulate the problem in the following sections.

\subsection{Assumptions}

The main assumptions of the proposed model are as follows:

- Customer demands are deterministic and can be met by multiple facilities;

- Facility disruption is independent and site-specific;

- The establishment cost is site-specific;

- A facility can be hardened at additional cost up to the highest level during its establishment;

- Only facilities hardened at level one are reliable and immune to failure;

- After a disruption event, based on a facility hardening level, the disrupted facility keeps serving part of its assigned demands, and unserved demands are reassigned to another reliable facility with a penalty cost per unit of demand;

- The transportation cost is related to the distance between a customer and its assigned facility per unit of demand.

\subsection{Mathematical model of reliable facility location-allocation with multi-level hardening}

The following parameters and variables are described and followed by the mathematical model.

\section{Notations and sets}

$I \quad$ Set of potential locations;

$J \quad$ Set of customers;

$L \quad$ Set of hardening levels;

$V \quad$ Penalty budget;

$B \quad$ Hardening budget;

$M \quad$ A large positive constant.

\section{Parameters}

$d_{j} \quad$ Demand of customer $j$;

$p_{i} \quad$ Failure probability of facility $i$;

$a_{i} \quad$ Establishment cost of facility $i$;

$s_{i} \quad$ Penalty cost of facility $i$

$c_{i} \quad$ Capacity of facility $i$;

$h_{i j} \quad$ Cost of per unit of demand to ship

from facility $i$ to customer $j$;

fil The lth level hardening cost of facility $i$.

Decision variables

$x_{i} \quad 1$ if plant $i$ is opened; 0, otherwise;

$z_{i l} \quad 1$ if plant $i$ is hardened at level $l ; 0$, otherwise;

$t t_{i j} \quad 1$ if facility $i$ is allocated to customer $j$; 0 , otherwise;

$x_{1 i j} \quad$ Fraction of customer $j$ 's demands is satisfied by facility $i$ as primary facility;

$x_{2 i r j} \quad$ Fraction of customer $j$ 's demands is not satisfied by facility $r$ as a primary facility, but is satisfied by a backup facility $i$.

\subsection{Mathematical model}

The mathematical model of the Reliable Facility Location-Allocation problem with Multi-level Harden- 
ing (RFLAMH) can be presented by:

$\min$ :

$$
\begin{aligned}
W= & \sum_{i} a_{i} \times x_{i}+\sum_{i} \sum_{j} x_{1 i j} \times z_{i 1} \times h_{i j} \\
& \times d_{j}+\sum_{i} \sum_{j} \sum_{l \geq 2} x_{1 i j} \times z_{i l} \times h_{i j} \\
& \times d_{j} \times\left(1-p_{i}\right)+\sum_{i} \sum_{j} x_{1 i j} \\
& \times\left(1-\sum_{l} z_{i l}\right) \times h_{i j} \times d_{j} \times\left(1-p_{i}\right) \\
& +\sum_{i} \sum_{j} \sum_{l \geq 2} x_{1 i j} \times z_{i l} \times p_{i} \times h_{i j} \\
& +\sum_{i} \sum_{l} f_{i l} \times z_{i l}, \\
& \times\left(\frac{d_{j}}{l}\right)_{i} \sum_{i} \sum_{j} \sum_{r \neq i} \sum_{l \geq 2} x_{2 i r j} \times x_{1 r j} \\
& \times \sum_{i} \sum_{j} \sum_{l \geq 2} x_{1 i j} \times p_{i} \times z_{i l} \times s_{i} \times d_{j} \\
& \times z_{r l} \times p_{r} \times h_{i j} \times d_{j} \times\left(\frac{l-1}{l}\right) \times s_{i} \times d_{j} \\
& +\sum_{i} \sum_{j} \sum_{r \neq i} x_{2 i r j} \times x_{1 r j} \\
& \times\left(1-\sum_{i} z_{r l} \sum_{j} x_{1 i j} \times p_{r} \times h_{i j} \times d_{j}\right.
\end{aligned}
$$

s.t.:

$$
\begin{array}{ll}
x_{1 i j} \leq x_{i} & \forall i, j, \\
x_{2 i r j} \leq x_{i} & \forall i, r \neq i, j, \\
\sum_{i} x_{1 i j}=1 & \forall j, \\
x_{2 i r j} \leq z_{i 1} & \forall i, r \neq i, j, \\
z_{i l} \leq x_{i} & \forall i, l,
\end{array}
$$

$$
\begin{aligned}
& \sum_{l} z_{i l} \leq 1 \quad \forall i, \\
& \sum_{i \neq r} x_{2 i r j}=t t_{r j} \times\left(1-z_{r 1}\right) \quad \forall r, j, \\
& t t_{i j} \geq x_{1 i j} \quad \forall i, j \\
& t t_{i j} \leq M \times x_{1 i j} \quad \forall i, j, \\
& \sum_{i} \sum_{j} \sum_{l \geq 2} x_{1 i j} \times z_{i l} \times s_{i} \times d_{j} \\
& \times\left(\frac{l-1}{l}\right)+\sum_{i} \sum_{j} x_{1 i j} \times\left(1-\sum_{l} z_{i l}\right) \\
& \times s_{i} \times d_{j} \leq V, \\
& \sum_{i} \sum_{l} f_{i l} \times z_{i l} \leq B \\
& \sum_{j} x_{1 i j} \times d_{j}+\sum_{j} \sum_{r \neq i} \sum_{l \geq 2} x_{2 i r j} \times x_{1 r j} \times z_{r l} \\
& \times d_{j} \times\left(\frac{l-1}{l}\right)+\sum_{j} \sum_{r \neq i} x_{2 i r j} \times x_{1 r j} \\
& \times\left(1-\sum_{l} z_{r l}\right) \times d_{j} \leq c_{i} \quad \forall i, \\
& x_{i}, z_{i l}, t t_{i j} \in\{0,1\} \quad \forall i, j, l, \\
& x_{1 i j}, x_{2 i r j} \in[0,1] \quad \forall i, r \neq i, j, l .
\end{aligned}
$$

Eq. (1) is the objective function that minimizes the total expected cost. Constraints (2) and (3) guarantee that only open facilities can serve as suppliers. Constraint (4) ensures that customer demand should be served completely. Constraint (5) ensures that only reliable facilities can serve customers as backup suppliers. Constraint (6) guarantees that only open facilities can be hardened at different levels. Constraint (7) limits facilities to being hardened at most to one level only. Constraints (8)-(10) guarantee that customers of failed facilities can be reassigned to backup facilities. Constraint (11) limits penalty budgets. Constraint (12) limits hardening budgets. Constraint (13) considers the capacity limitation of each facility to serve customers. Constraints (14) and (15) define decision variable types.

\subsection{Linearization of the proposed mathematical model}

It can be seen that the proposed model is nonlinear. Therefore, in order to linearize it, we introduce some 
Table 2. Categories of variables change and linearization.

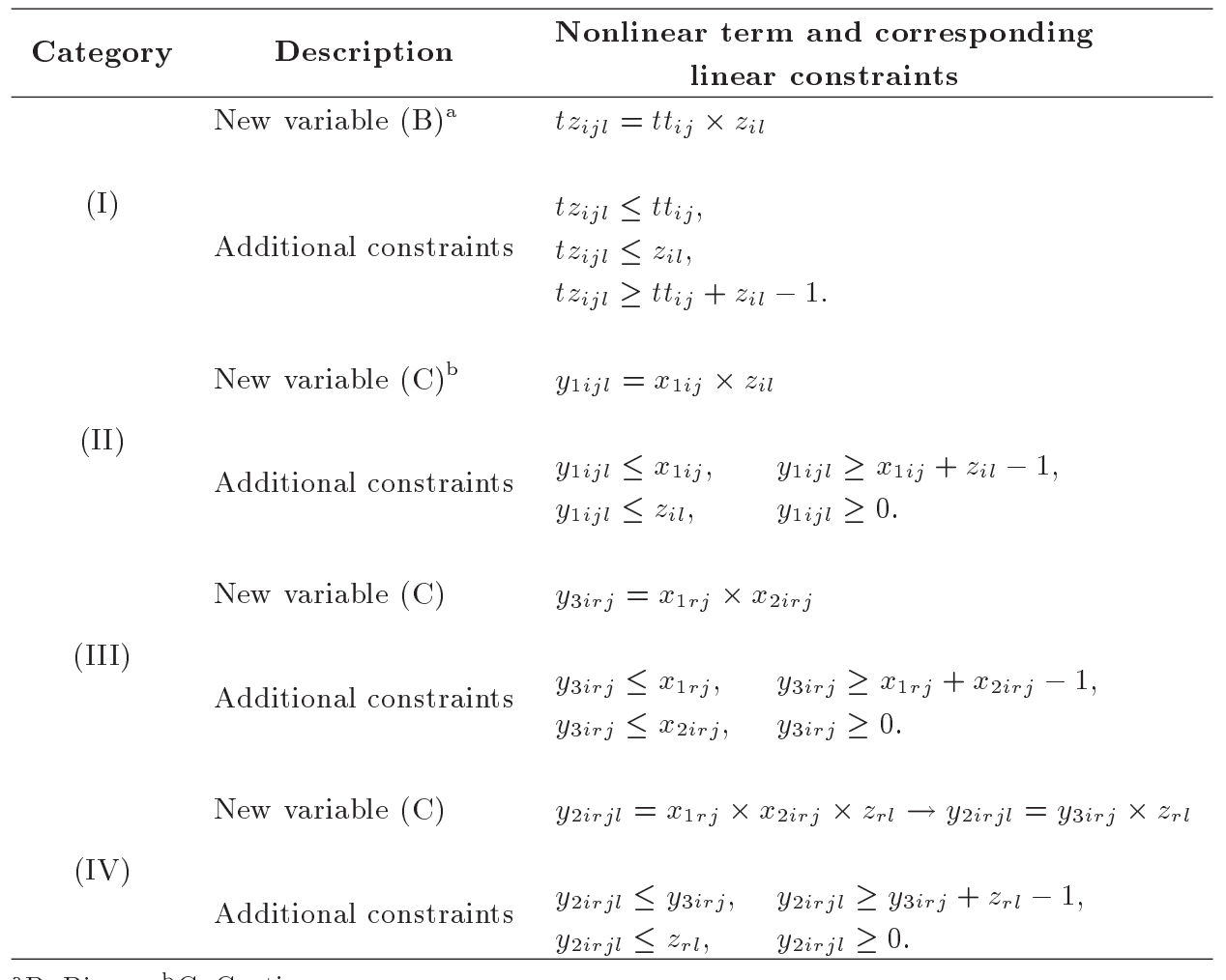

${ }^{\mathrm{a}} \mathrm{B}$ : Binary, ${ }^{\mathrm{b}} \mathrm{C}$ : Continuous.

new binary and continuous variables with additional constraints as reported in Table 2. For more detail, see $[17]$.

The equivalent linear model can be considered by: $\min$ :

$$
\begin{aligned}
W= & \sum_{i} a_{i} \times x_{i}+\sum_{i} \sum_{j} y_{1 i j 1} \times h_{i j} \times d_{j} \\
& +\sum_{i} \sum_{j} \sum_{l \geq 2} y_{1 i j l} \times h_{i j} \times d_{j} \times\left(1-p_{i}\right) \\
& +\sum_{i} \sum_{j} x_{1 i j} \times h_{i j} \times d_{j} \times\left(1-p_{i}\right) \\
& -\sum_{i} \sum_{j} \sum_{l} y_{1 i j l} \times h_{i j} \times d_{j} \times\left(1-p_{i}\right) \\
& +\sum_{i} \sum_{j} \sum_{l \geq 2} y_{1 i j l} \times p_{i} \times h_{i j} \times\left(\frac{d_{j}}{l}\right) \\
& +\sum_{i} \sum_{j} \sum_{r \neq i} \sum_{l \geq 2} y_{2 i r j l} \times p_{r} \times h_{i j} \times d_{j} \\
& \times\left(\frac{l-1}{l}\right)_{+} \sum_{i} \sum_{j} \sum_{r \neq i} y_{3 i r j} \times p_{r}
\end{aligned}
$$

$$
\begin{aligned}
& \times h_{i j} \times d_{j}-\sum_{i} \sum_{j} \sum_{r \neq i} \sum_{l} y_{2 i r j l} \\
& \times p_{r} \times h_{i j} \times d_{j}+\sum_{i} \sum_{j} \sum_{l \geq 2} y_{1 i j l} \\
& \times p_{i l} \times s_{i} \times d_{j} \times\left(\frac{l-1}{l}\right) \\
& +\sum_{i} \sum_{j} x_{1 i j} \times p_{i} \times s_{i} \times d_{j} \\
& -\sum_{i} \sum_{j} \sum_{l} y_{1 i j l} \times p_{i} \times s_{i} \times d_{j} \\
& +\sum_{i} \sum_{l} f_{i l} \times z_{i l},
\end{aligned}
$$

s.t.:

Constraints (2)-(7), (9), (10), (12), (14), (15):

$$
\begin{aligned}
& \sum_{i \neq r} x_{2 i r j}=t t_{r j}-t z_{r j 1} \quad \forall r, j, \\
& \sum_{i} \sum_{j} \sum_{l \geq 2} y_{1 i j l} \times s_{i} \times d_{j} \times\left(\frac{l-1}{l}\right)
\end{aligned}
$$




$$
\begin{aligned}
& +\sum_{i} \sum_{j} x_{1 i j} \times s_{i} \times d_{j}-\sum_{i} \sum_{j} \sum_{l} y_{1 i j l} \\
& \times s_{i} \times d_{j} \leq V \\
& \sum_{j} x_{1 i j} \times d_{j}+\sum_{j} \sum_{r \neq i} \sum_{l \geq 2} y_{2 i r j l} \times d_{j} \times\left(\frac{l-1}{l}\right) \\
& +\sum_{j} \sum_{r \neq i} y_{3 i r j} \times d_{j}-\sum_{j} \sum_{r \neq i} \sum_{l} y_{2 i r j l} \times d_{j} \\
& \leq c_{i}, \quad \forall i \\
& t z_{i j l} \leq t t_{i j} \quad \forall i, j, l, \\
& t z_{i j l} \leq z_{i l} \quad \forall i, j, l, \\
& t z_{i j l} \geq t t_{i j}+z_{i l}-1 \quad \forall i, j, l, \\
& y_{1 i j l} \leq x_{1 i j} \quad \forall i, j, l, \\
& y_{1 i j l} \leq z_{i l} \quad \forall i, j, l, \\
& y_{1 i j l} \geq x_{1 i j}+z_{i l}-1 \quad \forall i, j, l, \\
& y_{2 i r j l} \leq x_{2 i r j} \quad \forall i, r \neq i, j, \\
& y_{2 i r j l} \leq y_{1 r j l} \quad \forall i, r \neq i, j, l, \\
& y_{2 i r j l} \geq x_{2 i r j}+y_{1 r j l}-1 \quad \forall i, r \neq i, j, l, \\
& y_{3 i r j} \leq x_{1 r j} \quad \forall i, r \neq i, j, \\
& y_{3 i r j} \leq x_{2 i r j} \quad \forall i, r \neq i, j, \\
& y_{3 i r j} \geq x_{1 r j}+x_{2 i r j}-1 \quad \forall i, r \neq i, j, \\
& t z_{i j l} \in\{0,1\} \\
& \forall i, j, l, \\
& \forall i, r \neq i, j, l \text {. }
\end{aligned}
$$

\subsection{Solution method}

Since the classic solution algorithms for the capacitated location-allocation problem need a huge computational time, especially in large cases, it appears to be essential to propose an efficient solution approach to solving the model in large instances. On the other hand, the studies on location-allocation problems that utilize the Lagrangian relaxation-based solution method to solve the problem have presented the effectiveness of this method in solving such problems [3,9,10,18-31]. Herein, the Lagrangian Decomposition Algorithm (LDA) is proposed to solve the problem efficiently.

Solving the RFLAMH model is time consuming by the GAMS software, especially for large-scale problems. Because the proposed model contains binary and continuous variables, in this type of problems, a decomposition approach can intensively reduce computational times. In order to prepare the proposed model for decomposition in the first step by an implementation of linearization techniques, the proposed model is changed into a linear form, in which all terms in the objective function contain only one type of decision variables. In the next step, by relaxing constraints that connect binary and continuous variables, all terms in unrelaxed constraints contain only one type of decision variables. Then, the new linear relaxed problem can be decomposed easily into two sub-problems. The first one contains only binary variables that can be solved by the branch-and-bound algorithm. The second one contains only the continuous variables that can be solved by the simplex method in a short time. Numerical calculations and comparison in the next section show the efficiency of the proposed solution approach. The main structure of the LDA is described below:

1. Lagrangian relaxation: The Lagrangian relaxation problem is obtained by relaxing constraints that connect binary variables to continuous variables. The relaxed constraints appear in the new objective function by Lagrangian multipliers;

2. Decomposition: The relaxed problem is then decomposed into two independent sub-problems that can easily be solved. Both sub-problems can be solved optimally to generate lower and upper bounds concurrently;

3. Sub-gradient optimization: The Lagrangian multipliers are adjusted based on the violation of relaxed constraints, and the process is repeated until achieving a deterministic stopping criterion.

2.5.1. Lagrangian relaxation for the RFLAMH model In the RFLAMH model, by relaxing Constraints (2), (3), (5), (9), (10), (17), (24), and (25), the relaxed problem can be expressed by:

$\min$ :

$$
\begin{aligned}
W= & \sum_{i} a_{i} \times x_{i}+\sum_{i} \sum_{j} y_{1_{i j 1}} \times h_{i j} \times d_{j} \\
& +\sum_{i} \sum_{j} \sum_{l \geq 2} y_{1 i j l} \times h_{i j} \times d_{j} \times\left(1-p_{i}\right) \\
& +\sum_{i} \sum_{j} x_{1 i j} \times h_{i j} \times d_{j} \times\left(1-p_{i}\right) \\
& -\sum_{i} \sum_{j} \sum_{l} y_{1 i j l} \times h_{i j} \times d_{j} \times\left(1-p_{i}\right) \\
& +\sum_{i} \sum_{j} \sum_{l \geq 2} y_{1 i j l} \times p_{i} \times h_{i j} \times\left(\frac{d_{j}}{l}\right)
\end{aligned}
$$




$$
\begin{aligned}
& +\sum_{i} \sum_{j} \sum_{r \neq i} \sum_{l \geq 2} y_{2 i r j l} \times p_{r} \times h_{i j} \times d_{j} \\
& \times\left(\frac{l-1}{l}\right)+\sum_{i} \sum_{j} \sum_{r \neq i} y_{3 i r j} \times p_{r} \\
& \times h_{i j} \times d_{j}-\sum_{i} \sum_{j} \sum_{r \neq i} \sum_{l} y_{2 i r j l} \times p_{r} \\
& \times h_{i j} \times d_{j}+\sum_{i} \sum_{j} \sum_{l \geq 2} y_{1 i j l} \times p_{i} \\
& +\sum_{i} \sum_{j} \sum_{l} u_{7 i j l} \times\left(y_{1 i j l}-z_{i l}\right) \\
& +\sum_{i} \times d_{j} \times\left(\frac{l-1}{l}\right)+\sum_{i} \sum_{j} x_{1 i j} \times\left(x_{1 i j}+z_{i l}-1-y_{1 i j l}\right) \\
& +\sum_{i j i} \times\left(t t_{i j}-\sum_{j} u_{5 i j} \times\left(x_{1 i j}-t t_{i j}\right)\right. \\
& \left.+p_{i} \times x_{1 i j}\right) \\
& +\sum_{i} \sum_{r \neq i} \sum_{j} \sum_{j} u_{2 i r j} \times\left(x_{2 i r j}-x_{j} u_{3 i r j} \times\left(x_{2 i r j}-z_{i 1}\right)\right. \\
& +\sum_{i} \sum_{j} \sum_{l} y_{1 i j l} u_{1 i j} \times\left(\sum_{i \neq r} x_{2 i r j}-\left(t t_{r j}-t z_{r j 1}\right)\right. \\
& \times p_{i} \times \times s_{i} \times d_{j}+\sum_{i} \sum_{l} f_{i l} \times z_{i l}
\end{aligned}
$$

s.t.:

Constraints (4), (6), (7), (12), (14), (15), (18), (19), (23), (26)-(33).

\subsubsection{Decomposition of the relaxed RFLAMH model} The relaxed problem is decomposed into two independent sub-problems: location-hardening and allocation sub-problems, called sub1 and sub2, respectively. In sub-problems, $u_{i j}, u_{i j l}$, and $u_{i r j}$ are Lagrangian multi- pliers.

\section{Sub-problem 1 (sub1):}

The first sub-problem (sub1) that includes binary variables is given only by:

$$
\begin{aligned}
\min : & \\
W= & \sum_{i} a_{i} \times x_{i}+\sum_{i} \sum_{l} f_{i l} \times z_{i l} \\
& -\sum_{i} \sum_{j} u_{1 i j} \times x_{i} \\
& -\sum_{i} \sum_{r \neq i} \sum_{j} u_{2 i r j} \times x_{i} \\
& -\sum_{i} \sum_{r \neq i} \sum_{j} u_{3 i r j} \times z_{i 1} \\
& -\sum_{r} \sum_{j} u_{4 r j} \times\left(t t_{r j}-t z_{r j 1}\right) \\
& -\sum_{i} \sum_{j} u_{5 i j} \times t t_{i j}+\sum_{i} \sum_{j} u_{6 i j} \\
& \times t t_{i j}-\sum_{i} \sum_{j} \sum_{l} u_{7 i j l} \times z_{i l} \\
& +\sum_{j} \sum_{l} u_{8 i j l} \times z_{i l},
\end{aligned}
$$

s.t.:

Constraints (6), (7), (12), (14), (20)-(22), and (32).

\section{Sub-problem 2 (sub2):}

The second sub-problem (sub2) that includes continuous variables only is given by:

$\min$ :

$$
\begin{aligned}
W_{\text {sub } 2}= & \sum_{i} \sum_{j} y_{1 i j 1} \times h_{i j} \times d_{j} \\
& +\sum_{i} \sum_{j} \sum_{l \geq 2} y_{1 i j l} \times h_{i j} \times d_{j} \times\left(1-p_{i}\right) \\
& +\sum_{i} \sum_{j} x_{1 i j} \times h_{i j} \times d_{j} \times\left(1-p_{i}\right) \\
& -\sum_{i} \sum_{j} \sum_{l} y_{1 i j l} \times h_{i j} \times d_{j} \times\left(1-p_{i}\right) \\
& +\sum_{i} \sum_{j} \sum_{l \geq 2} y_{1 i j l} \times p_{i} \times h_{i j} \times\left(\frac{d_{j}}{l}\right)
\end{aligned}
$$




$$
\begin{aligned}
& +\sum_{i} \sum_{j} \sum_{r \neq i} \sum_{l \geq 2} y_{2 i r j l} \times p_{r} \times h_{i j} \times d_{j} \\
& \times\left(\frac{l-1}{l}\right)+\sum_{i} \sum_{j} \sum_{r \neq i} y_{3 i r j} \times p_{r} \\
& \times h_{i j} \times d_{j}-\sum_{i} \sum_{j} \sum_{r \neq i} \sum_{l} y_{2 i r j l} \times p_{r} \\
& \times h_{i j} \times d_{j}+\sum_{i} \sum_{j} \sum_{l \geq 2} y_{1 i j l} \times p_{i} \times s_{i} \\
& \times d_{j} \times\left(\frac{l-1}{l}\right)+\sum_{i} \sum_{j} x_{1 i j} \times p_{i} \times \times s_{i} \\
& \times d_{j}-\sum_{i} \sum_{j} \sum_{l} y_{1 i j l} \times p_{i} \times \times s_{i} \times d_{j} \\
& +\sum_{i} \sum_{j} u_{1 i j} \times x_{1 i j}+\sum_{i} \sum_{r \neq i} \sum_{j} u_{2 i r j} \\
& \times x_{2 i r j}+\sum_{i} \sum_{r \neq i} \sum_{j} u_{3 i r j} \times x_{2 i r j} \\
& +\sum_{r} \sum_{j} u_{4 r j} \times \sum_{i \neq r} x_{2 i r j}+\sum_{i} \sum_{j} u_{5 i j} \\
& \times x_{1 i j}-\sum_{i} \sum_{j} u_{6 i j} \times M \times x_{1 i j} \\
& +\sum_{i} \sum_{j} \sum_{l} u_{7 i j l} \times y_{1 i j l} \\
& +\sum_{i} \sum_{j} \sum_{l} u_{8 i j l} \times\left(x_{1 i j}-y_{1 i j l}-1\right),
\end{aligned}
$$

s.t.:

Constraints (4), (15), (18), (19), (22), (26)-(31), and (33).

\subsubsection{Sub-gradient optimization}

In order to solve the decomposed problems, a subgradient algorithm is used to calculate the Lagrangian multipliers. For more detail, see $[29,30]$. The following notations and steps are used for the procedure.

\section{Notations}

$W_{L B} \quad$ Best lower bound

$W_{U B} \quad$ Best upper bound

$£_{M} \quad$ Maximum of the relative gap

$L \quad$ Step size

$\mu \quad$ Step size coefficient

$t \quad$ Number of iterations
$t_{\max } \quad$ Max iteration
$\varepsilon_{q}^{t} \quad$ Violation of constraint $q$ for a solution in iteration $t$
$I M_{0} \quad$ Max iteration without improvement

In order to generate lower and upper bounds concurrently with the suitable quality, sub-gradient optimization is applied by using the following steps:

Step 0: Initialize the parameters $\left(u_{q}, \mu, t_{\max }, I M_{0}\right.$, $\left.£_{M}\right)$

1. Set $W_{L B} \rightarrow-\infty$ and $W_{U B} \rightarrow+\infty$.

Step 1: 1. Solve sub1,

2. Solve sub2,

3. Set $W_{L R}^{*}=W_{s u b 1}^{*}+W_{s u b 2}^{*}$,

4. If $W_{L R}^{*} \geq W_{L B}$, set $W_{L B}=W_{L R}^{*}$, otherwise, $I M=I M+1$,

5. Fix sub1 solution in the main problem and get $\left(W^{*}\right)$,

6. If $\left(W^{*}<W_{U B}\right) \rightarrow$ Set $W_{U B}=W^{*}$,

7. Set $£=\frac{\left(W_{U B}-W_{L B}\right)}{W_{L B}}$.

Step 2: 1. If $\left(t \geq t_{\max }\right.$ OR $\left.£ \leq £_{M}\right)$, stop, otherwise, go to the next step.

Step 3: 1. If $I M \geq I M_{0}$, then $\mu=\mu / 2$,

2. Set $L=\mu \times \frac{\left(W_{U B}-W_{L B}\right)}{\sum_{q}\left(\varepsilon_{q}^{t}\right)^{2}}$

3. Set $u_{q}^{t+1}=\max \left(0, u_{q}^{t}+\left(L \times \varepsilon_{q}^{t}\right)\right)$,

4. Set $t \leftarrow t+1$ and return to Step 1 .

\section{Computational results}

Some numerical analyses from different aspects are described in two parts. In the first one, the necessity of the proposed mathematical model (RFLAMH) is evaluated by comparing it to the classical hardening facility location-allocation model ( $\mathrm{RFLACH})$. Then, in the next part, the efficiency of the proposed solution algorithm (LDA) is considered in both solution quality and computational time.

\subsection{The proposed model's effect in the network design compared to classic models} To consider the effectiveness of the proposed mathematical model in a network design, the behavior of the proposed model is compared with the classical hardening facility location-allocation model (RFLACH) in an instance. The selected instance consists of 15 facilities, 20 customers, and 3 hardening levels in different values of failure probability. The Objective Function Values (OFVs) of the two models are obtained for selected instance, considering various degrees of failure probabilities. Figure 1 illustrates the difference of two OFVs by increasing the failure probability. 


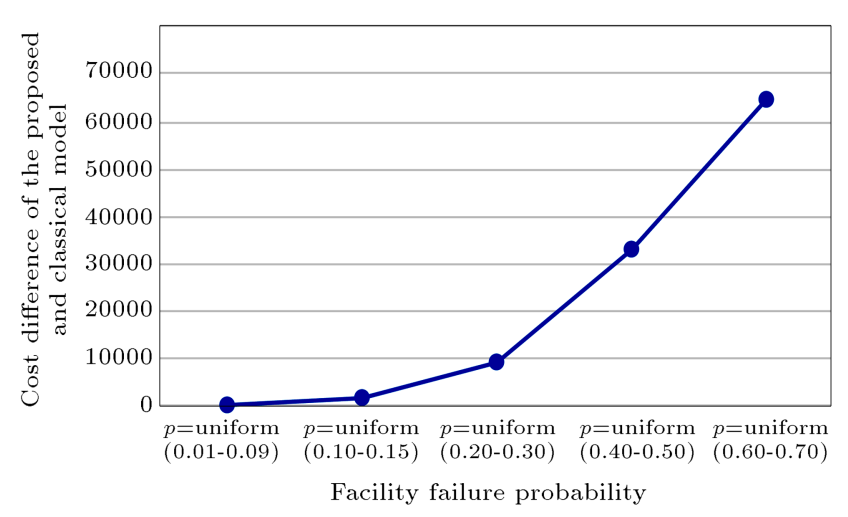

Figure 1. Difference of OFVs of RFLAMH and RFLACH in different failure probabilities.

The comparison of results shows that the total cost of the RFLAMH model is lower than that of the RFLACH model. This comparison confirms the necessity of using the proposed model in the network design. The numerical analysis confirms that the proposed model should be used in cases with a large value of failure probabilities of facilities.

\subsection{Evaluating the efficiency of $L D A$}

In this section, the performance of the proposed solution approach is evaluated using some test problems with several sizes. For mentioned problems, different parameters (e.g., capacity, facility establishment cost, facilities failure probability, facility hardening cost, and penalty cost) are randomly generated by the uniform distribution function, as presented in Table 3 . The results of the LDA in each instance are compared with the obtained results of solving the linear proposed model by the CPLEX solver in GAMS 24.7.4. The best values of the LDA upper bounds and the gaps of the LDA compared to the CPLEX are given for each problem in Table 4. For each problem, the LDA gap in comparison to the CPLEX is obtained by:

$$
R G_{2}=\frac{\left(\mathrm{Upper} \text { Bound }_{\mathrm{LDA}}-\mathrm{OFV}_{\mathrm{CPLEX}}\right)}{\mathrm{OFV}_{\mathrm{CPLEX}}}
$$

Table 3. Parameters for the generated test problems and initial multipliers for the LDA.

\begin{tabular}{ll}
\hline \multicolumn{1}{c}{ Parameters } & \multicolumn{1}{c}{ Multipliers } \\
\hline$a_{i}=$ uniform $[0.01,0.1]$ & $u_{1 i j}=$ uniform $(0.01,0.1)$ \\
$c_{i}=$ uniform[10000,13000] & $u_{2 i r j}=$ uniform $(0.01,0.1)$ \\
$d_{j}=$ uniform $[200,500]$ & $u_{3 i r j}=$ uniform $(0.01,0.1)$ \\
$h_{i j}=$ uniform $[10,15]$ & $u_{4 r j}=$ uniform $(0.01,0.1)$ \\
$p_{i}=$ uniform $[0.01,0.1]$ & $u_{5 r j}=$ uniform $(0.01,0.1)$ \\
$s_{i}=$ uniform $[20,30]$ & $u_{6 r j}=0$ \\
$f_{i 1}=0.01 *$ uniform $[30,50]$ & $u_{7 i j l}=$ uniform $(0.01,0.1)$ \\
$f_{i 2}=0.01 *$ uniform $[15,20]$ & $u_{8 i j l}=0$ \\
$f_{i 3}=0.1 *$ uniform $[12,15]$ & \\
\hline
\end{tabular}

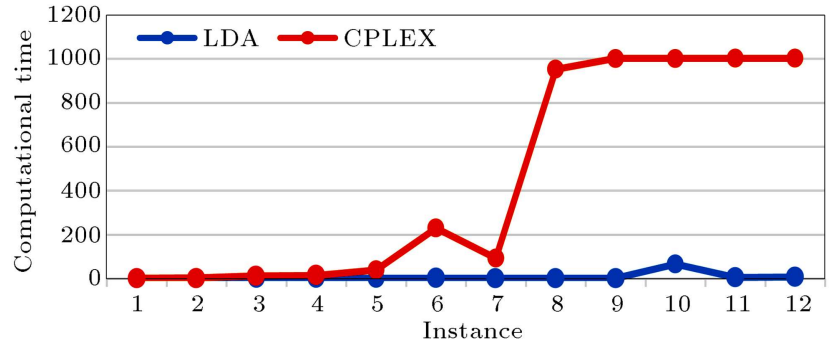

Figure 2. Comparison of the LDA and CPLEX computational times.

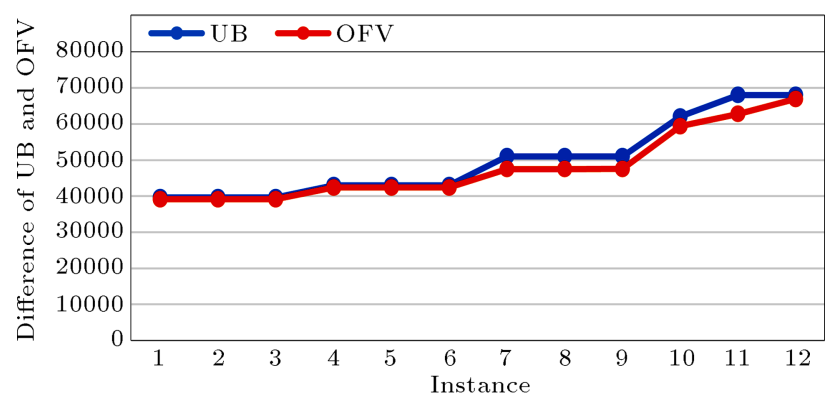

Figure 3. Comparison of the upper bound and OFV.

It is expected that the proposed solution algorithm be more efficient in solving the RFLAMH compared to the classic solution approaches. Results of a comparison are depicted in Figures 2 and 3.

\section{Conclusion and recommendations}

In the real world, facilities can be disrupted in some cases. Facility disruption may lead to the increase of network costs and customers dissatisfaction. This concept can be more important for cases with higher penalty costs of unserved demands. For example, telecommunication networks, relief distribution centers, and emergency rooms in healthcare services are some examples of such applications. The facilities should be hardened enough to prevent a failure. In this paper, a mathematical model was proposed, and a static resilient network was considered. The numerical studies confirmed that the network designed by the proposed model led to a significantly less cost compared to the network designed by classic models. The difference between the proposed model and previous classic models had a considerable difference in cases with high facility failure probabilities. The human-based monitoring cases are some examples of such cases. Since solving location-allocation models is time consuming by the classical solution algorithms, especially for largescale problems, Lagrangian Decomposition Algorithm (LDA) was one of the solution approaches to this problems. In order to decompose the problem, the proposed non-linear model was linearized. The numerical results confirmed that the LDA had the ability 
Table 4. Computational results of the LDA and CPLEX.

\begin{tabular}{|c|c|c|c|c|c|c|c|c|c|}
\hline \multirow{2}{*}{ Instance } & \multicolumn{3}{|c|}{ Problem size } & \multicolumn{3}{|c|}{ CPLEX } & \multicolumn{3}{|c|}{ LDA } \\
\hline & $I^{\mathrm{a}}$ & $J^{\mathrm{b}}$ & $L^{\mathrm{c}}$ & $\mathrm{OFV}^{\mathrm{d}}$ & $R G_{1}^{\mathrm{e}}$ & CPU $^{f}$ (Sec.) & $\mathrm{UB}^{\mathrm{g}}$ & $R G_{2}$ & CPU (Sec.) \\
\hline 1 & 5 & 10 & 2 & 39171.390 & 0 & 1.419 & 39681.224 & 0.013 & 0.927 \\
\hline 2 & 5 & 10 & 3 & 39171.390 & 0 & 4.767 & 39681.224 & 0.013 & 0.940 \\
\hline 3 & 5 & 10 & 4 & 39171.390 & 0 & 12.280 & 39681.224 & 0.013 & 1.184 \\
\hline 4 & 7 & 13 & 2 & 42430.504 & 0 & 14.651 & 43038.358 & 0.014 & 1.244 \\
\hline 5 & 7 & 13 & 3 & 42430.504 & 0 & 40.199 & 43038.358 & 0.014 & 1.354 \\
\hline 6 & 7 & 13 & 4 & 42430.504 & 0 & 230.409 & 43038.358 & 0.014 & 1.403 \\
\hline 7 & 10 & 15 & 2 & 47505.120 & 0 & 93.728 & 51069.423 & 0.075 & 2.063 \\
\hline 8 & 10 & 15 & 3 & 47505.120 & 0 & 952.693 & 51069.423 & 0.075 & 2.264 \\
\hline 9 & 10 & 15 & 4 & 47552.983 & 0.25 & 1001.228 & 51069.423 & 0.074 & 2.596 \\
\hline 10 & 15 & 20 & 2 & 59434.369 & 1 & 1001.897 & 62102.627 & 0.045 & 66.827 \\
\hline 11 & 15 & 20 & 3 & 62818.390 & 0.85 & 1002.393 & 68054.859 & 0.083 & 5.779 \\
\hline 12 & 15 & 20 & 4 & 66966.662 & 1 & 1002.956 & 68054.859 & 0.016 & 7.263 \\
\hline \multirow{2}{*}{\multicolumn{4}{|c|}{$\begin{array}{l}{ }^{\mathrm{a}} I: \text { Number of facilities; } \\
\mathrm{d} \text { OFV: Objective Function Value; } \\
\text { gUB: Upper Bound of LDA. }\end{array}$}} & \multirow{2}{*}{\multicolumn{5}{|c|}{$\begin{array}{ll}{ }^{\mathrm{b}} J \text { : Number of customers; } & { }^{\mathrm{c}} L: \text { Hardening levels; } \\
{ }^{\mathrm{e}} R G_{1} \text { : CPLEX relative gap; } & { }^{\mathrm{f}} \text { CPU: Computational time (sec.); }\end{array}$}} & \\
\hline & & & & & & & & & \\
\hline
\end{tabular}

to generate very near-optimal solutions to the exact ones in less computation time. There are some cases with uncertain customer demands; therefore, demand uncertainty can be one of the directions for future researches. A decision about different transportation modes with different environmental pollutions in the problem modeling can be made in order to reduce the total expected cost of location, hardening, transportation, and decreasing of environmental pollutions. Using other decomposition algorithms can be considered as another future work.

\section{References}

1. Amoaning, Y. "A resiliency framework for planning in state transportation agencies", Gor. Ins. Technol., pp. 1-2 (2013).

2. Azad, N., Davoudpour, H., Saharidis, G.K.D., and Shiripour, M. "A new model to mitigating random disruption risks of facility and transportation in supply chain network design", International Journal of Advanced Manufacturing Technology, 69, pp. 1757-1774 (2013).

3. Qingwei, L. and Savachkin, A. "A heuristic approach to the design of fortified distribution networks", Trans portation Research Part E, 50, pp. 138-148 (2013).

4. Church, R.L., Middleton, R.S., and Scaparra, M.P. "Identifying critical infrastructure: The median and covering facility interdiction problems", Annals of the Association of American Geographers, 94, pp. 491-502 (2004).

5. Scaparra, M.P. and Church, R.L. "A bilevel mixedinteger program for critical infrastructure protection planning", Computer and Operations Research, 35, pp. 1905-1923 (2008).
6. Aksen, D., Necati, A., and Piyade, N. "The budget constrained r-interdiction median problem with capacity expansion", Central European Journal of Operations Research, 18, pp. 269-291 (2010).

7. Daskin, M., Liberatore, F., and Scaparra, M.P. "Hedging against disruptions with ripple effects in location analysis", OMEGA, 40, pp. 21-30 (2012).

8. Medal, H.R., Pohl, E.A., and Rossetti, M.D. "A multiobjective integrated facility location-hardening model: Analyzing the pre and post-disruption tradeoff", $E u$ ropean Journal of Operational Research, 237, pp. 257270 (2014).

9. Snyder, L. and Daskin, M.S. "Reliability models for facility location: the expected failure cost case", Transportation Science, 39, pp. 400-416 (2005).

10. Lim, M., Daskin, M.S., Bassamboo, A., and Chopra, S. "Facility reliability problem: formulation, properties, and algorithm", Naval Research Logistics, 57, pp. 5870 (2009).

11. Shishebori, D., Snyder, L.V., and Jabalameli, M.S. "A reliable budget-constrained facility location/network design problem with unreliable facilities", Networks and Spatial Economics, 14, pp. 549-580 (2014).

12. Mohammadi, M., Tavakkoli-Moghaddam, R., Siadat, A., and Dantan, J.-Y. "Design of a reliable logistics network with hub disruptions under uncertainty", Applied Mathematical Modelling, 40, pp. 5621-5642 (2016).

13. Fattahi, M., Govindan, K., and Keyvanshokooh, E. "Responsive and resilient supply chain network design under operational and disruption risks with delivery lead-time sensitive customers", Transportation Research Part E: Logistics and Transportation Review, 101, pp. 176-200 (2017). 
14. Jabbarzadeh, A., Fahimnia, B., Sheu, J.B., and Shahmoradi-Moghadam, H. "Designing a supply chain resilient to major disruptions and supply/demand interruptions", Transportation Research Part B, 94, pp. 121-149 (2016).

15. Losada, C., Scaparra, M.P., and O'Hanley, J.R. "Optimizing system resilience: a facility protection model with recovery time", European Journal of Operational Research, 217, pp. 519-530 (2012).

16. Rastgoufard, P., Leevongwat, I., and Rastgoufard, R. "Electric grid hardening and resiliency: part I, resiliency and safety", Engineering \& Technology Reference, 14 pages (2016).

17. Niakan, F. and Rahimi, M. "A multi-objective healthcare inventory routing problem: A fuzzy possibilistic approach", Transportation Research Part E: Logistics and Transportation Review, 80, pp. 74-94 (2015).

18. Holmberg, K., Ronnqvist, M., and Yuan, D. "An exact algorithm for the capacitated facility location problems with single sourcing", European Journal of Operational Research, 113, pp. 544-559 (1999).

19. Barcelo, J. and Casanovas, J. "A heuristic Lagrangean algorithm for the capacitated plant location problem", European Journal of Operational Research, 15, pp. 212-226 (1984).

20. Beasley, J.E. "Lagrangean heuristics for location problems", European Journal of Operational Research, 65, pp. 383-399 (1993).

21. Agar, M.C. and Salhi, S. "Lagrangean heuristics applied to a variety of large capacitated plant location problems", Journal of the Operational Research Society, 49(1), pp. 1072-1084 (1998).

22. Cortinhal, M.J. and Captivo, M.E. "Upper and lower bounds for the single source capacitated location problem", European Journal of Operational Research, 151, pp. 333-351 (2003).

23. Chen, C.H. and Ting, C.J. "Combining Lagrangian heuristic and ant colony system to solve the single source capacitated facility location problem", Transportation Research Part E: Logistics and Transportation Review, 44(6), pp. 1099-1122 (2008).

24. Jena, S.D., Cordeau, J.F., and Gendron, B. "Solving a dynamic facility location problem with partial closing and reopening", Computers and Operations Research, 67, pp. 143-154 (2016).

25. Rönnqvist, M., Tragantalerngsak, S., and Holt, J. "A repeated matching heuristic for the single-source capacitated facility location problem", European Journal of Operational Research, 116, pp. 51-68 (1999).

26. Wu, T., Chu, F., Yang, Z., and Zhou, Z. "A Lagrangean relaxation approach for a two-stage capacitated facility location problem with choice of facility size", In Proceedings of the IEEE International Conference on Systems, Man, and Cybernetics, pp. 713-718 (2015).
27. Li, Q., Savachkin, A., and Zeng, B.O. "Reliable facility location design under disruptions", Computers and Operations Research, 40, pp. 901-909 (2013).

28. Li, Q. and Savachkin, A. "Reliable distribution networks design with nonlinear fortification function", International Journal of Systems Science, 47, pp. 805813 (2014).

29. Held, M., Crowder, D., and Wolf, H. "Validation of subgradient optimization", Mathematical Programming, 6, pp. 62-88 (1974).

30. Eskandarzadeh, S., Tavakkoli-Moghaddam, R., and Azaron, A. "An extension of the relaxation algorithm for solving a special case of capacitated arc routing problems", Journal of Combinatorial Optimization, 17(2), pp. 214-234 (2009).

31. Shishebori, D., Yousefi Babadi, A., and Noormohammadzadeh, Z.A. "Lagrangian relaxation approach to fuzzy robust multi-objective facility location network design problem", Scientia Iranica, 25, pp. 1750-1767 (2018).

\section{Biographies}

Zahra Esfandiyari is currently a $\mathrm{PhD}$ student under the supervision of Professor Bashiri at Islamic Azad University-Science \& Research Branch in Tehran. She obtained her BSc in Pure Mathematics from Iran University of Alzahra in Tehran. She obtained her MSc in Industrial Engineering from the Islamic Azad University Science \& Research Branch in Tehran. Her research interests include supply chain, disruption and mathematical modeling.

Mahdi Bashiri is a Professor of Industrial Engineering at Shahed University. He holds a BSc degree in Industrial Engineering from Iran University of Science and Technology as well as MSc and PhD degrees from Tarbiat Modarres University. $\mathrm{He}$ is a recipient of the 2013 young national top scientist award from the Academy of Sciences of the Islamic Republic of Iran. He serves as the Editor-in-Chief of Journal of Quality Engineering and Production Optimization published in Iran and the Editorial Board member of some reputable academic journals. His research interests are facility planning, stochastic optimization, meta-heuristics, and multi-response optimization. He published about 10 books and more than 190 papers in reputable academic journals and conferences.

Reza Tavakkoli-Moghaddam is a Professor of Industrial Engineering at the College of Engineering, University of Tehran in Iran. He obtained his $\mathrm{PhD}, \mathrm{MSc}$, and BSc degrees in Industrial Engineering from Swinburne University of Technology in Melbourne (1998), University of Melbourne in Melbourne (1994), and Iran University of Science and Technology in Tehran 
(1989), respectively. He serves as the Editor-in-Chief of Journal of Industrial Engineering published in Iran. He is a recipient of the 2009 and 2011 Distinguished Researcher Awards and the 2010 and 2014 Distinguished Applied Research Awards at University of Tehran, Iran. He has been selected as the National Iranian
Distinguished Researcher in 2008 and 2010 by the Ministry of Science, Research, and Technology in Iran. He has obtained the outstanding rank as the top $1 \%$ scientist and researcher in the world elite group since 2015. He has published 4 books, 22 book chapters, and more than 1000 journal and conference papers. 